\title{
VALIDAÇÃO DE TECNOLOGIAS EDUCACIONAIS: ESTUDO BIBLIOMÉTRICO EM TESES E DISSERTAÇÕES DE ENFERMAGEM
}

\author{
VALIDATION OF EDUCATIONAL TECHNOLOGIES: BIBLIOMETRIC STUDY IN NURSING THESES AND \\ DISSERTATIONS
}

\section{VALIDACIÓN DE TECNOLOGÍAS EDUCATIVAS: ESTUDIO BIBLIOMÉTRICO EN TESIS Y DISERTACIONES DE ENFERMERÍA}

Alexandra do Nascimento Cassiano ${ }^{1}$, Carlos Jordão De Assis Silva², Isadora Lorenna Alves Nogueira ${ }^{3}$, Tatiana Maria Nóbrega Elias ${ }^{4}$, Elizabeth Teixeira ${ }^{5}$, Rejane Maria Paiva de Menezes $^{6}$

\section{RESUMO}

Objetivo: Analisar as estratégias metodológicas utilizadas para a validação de tecnologias educacionais em teses e dissertações de enfermagem no Brasil. Método: Trata-se de um estudo documental bibliométrico. A busca foi realizada no catálogo de Teses e Dissertações da Coordenação de Aperfeiçoamento de Pessoal de Nível Superior. O mapeamento considerou o perfil das produções e das tecnologias educacionais, as estratégias de validação e a aplicação da lei de Zipt. A análise foi realizada com o Statistical Package for the Social Sciences 21.0 e o IRaMUTeQ 0.7 alpha 2. Resultados: Obtiveram-se 156 produções. O perfil das produções foi de dissertações de mestrado acadêmico, consideradas estudos metodológicos, estando a maioria desenvolvida na região Nordeste do País. As tecnologias educacionais corresponderam a materiais impressos ou audiovisuais, direcionados à comunidade e à área de enfermagem médico-cirúrgica. O processo de validação ocorreu, em sua maioria por concordância, com participação de juízes especialistas e da população-alvo, com uso de instrumentos validados e aplicação do Índice de Validação de Conteúdo. A partir da aplicação da Lei de Zipt, destacaram-se validação, juiz e estudo metodológico. Conclusão: O mapeamento propiciou a visibilidade da produção científica, indicando um conhecimento com distribuição desigual no que tange à região onde se produz tal capital intelectual.

Descritores: Estudo de Validação; Tecnologia educacional; Enfermagem; Bibliometria; Dissertação Acadêmica.

\begin{abstract}
Objective: To analyze the methodological strategies used for the validation of educational technologies in nursing theses and dissertations in Brazil. Method: This is a bibliometric documentary study. The search was carried out in the catalog of Theses and Dissertations of the Coordination for the Improvement of Higher Education Personnel. The mapping considered the profile of productions and educational technologies, validation strategies and the application of the Zipt's law. The analysis was performed in the Statistical Package for the Social Sciences 21.0 and IRaMuTeQ 0.7 alpha 2. Results: A total of 156 productions were obtained. The profile of the productions was of academic master's dissertations, considered methodological studies, mostly developed in the Northeast region of the country. The educational technologies corresponded to printed or audiovisual materials directed to the community and the medical-surgical nursing area. The validation process took place, mostly by agreement, with the participation of expert judges and the target population, using validated instruments and applying the Content Validation Index. With the application of the Zipt's Law, validation, judge and methodological study stood out. Conclusion: The mapping provided the visibility of the scientific production, indicating knowledge with an unequal distribution regarding the region where such intellectual capital is produced.

Descriptors: Validation Study; Educational technology; Nursing; Bibliometric; Academic Dissertation.
\end{abstract}

\section{RESUMEN}

Objetivo: Analizar las estrategias metodológicas utilizadas para validar las tecnologías educativas en tesis y disertaciones de enfermería en Brasil. Método: Este es un estudio bibliométrico documental. La búsqueda se realizó en el catálogo de Tesis y Disertaciones de la Coordinación para la Mejora del Personal de Educación Superior. El mapeo consideró el perfil de las producciones y tecnologías educativas, las estrategias de validación y la aplicación de la ley de Zipt. El análisis se realizó con el Paquete Estadístico para Ciencias Sociales 21.0 e IRaMuTeQ 0.7 alpha 2. Resultados: Se obtuvieron 156 producciones. El perfil de las producciones fue de disertaciones de maestría académica, consideradas estudios metodológicos, la mayoría de los cuales se desarrolló en la región noreste del país; las tecnologías educativas correspondían a materiales impresos o audiovisuales, dirigidos a la comunidad y en el área de enfermería médico-quirúrgica; El proceso de validación se realizó principalmente mediante un acuerdo con la participación de jueces expertos y la población objetivo, utilizando instrumentos validados y aplicando el Índice de Validación de Contenido. De la aplicación de la Ley Zipt, se destacó la validación, el juez y el estudio metodológico. Conclusión: El mapeo proporcionó la visibilidad de la producción científica indicando conocimiento con una distribución desigual con respecto a la región donde se produce dicho capital intelectual.

Descriptores: Estudio de Validación; Tecnología Educacional; Enfermería; Bibliometría; Tesis Académica.

'Doutoranda em Enfermagem pela Universidade Federal do Rio Grande do Norte - UFRN. ${ }^{2}$ Doutorando em Enfermagem pela UFRN. ${ }^{3}$ Doutoranda em Enfermagem pela UFRN. ${ }^{4}$ Doutoranda em Enfermagem pela UFRN. ${ }^{5}$ Pós-doutorado em Sociologia pela Universidade de Coimbra. Pós-doutorado Senior em Enfermagem pela Universidade do Estado do Rio de Janeiro. Docente Permanente do Mestrado Profissional de Enfermagem em Saúde Pública da Universidade do Estado do Amazonas e do Mestrado Profissional em Saúde da Família da Universidade do Estado do Amazonas. ${ }^{6}$ Doutora em Enfermagem. Docente da graduação e pós-graduação em Enfermagem da UFRN.

\section{Como citar este artigo:}

Cassiano AN, Silva CJA, Nogueira ILA, et al. Validação de tecnologias educacionais: estudo bibliométrico em teses e dissertações de enfermagem. Revista de Enferamgem do Centro-Oeste Mineiro. 2020;10:e3900. [Access http://doi.org/10.19175/recom.v10i0.3900

]; Available in: DOI: 


\section{INTRODUÇÃO}

As Tecnologias Educacionais (TE) são ferramentas que contribuem para a ampliação do conhecimento, das habilidades, das atitudes e do autoconhecimento necessários para assumir a responsabilidade relacionada às práticas de ensino e do cuidado. Essas são a base para potencializar e empoderar a autonomia dos sujeitos, comunidade, estudantes e profissionais de saúde ${ }^{(1-2)}$. Em sua maioria, as TE são consideradas como tecnologias leves, que se caracterizam como tipos de produção de vínculo, de acolhimento e de gestão de processos de trabalho que permeiam as relações interpessoais ${ }^{(3)}$.

Em resultados de uma análise do conceito $T E$, identificaram-se, como atributos do constructo, as características da ferramenta de apoio: aquisitiva de competências, mediadora de conhecimentos, promotora da saúde, útil para o cuidado e interativa. Como consequentes, para os que a utilizam, tem-se a melhoria na qualidade de vida, no desenvolvimento de competências, na satisfação dos cuidados, na adoção de comportamentos saudáveis, no estímulo à reflexão, no favorecimento do vínculo e da capacidade criativa, na promoção da adesão, na minimização da ansiedade, na uniformização das informações e na promoção do empoderamento ${ }^{(4)}$.

$\mathrm{Na}$ enfermagem, as tecnologias voltadas à educação se expressam por meio de três tendências: tecnologias para a educação técnica e superior, com estudantes; tecnologias para a educação em saúde, com a comunidade; e tecnologias para a educação continuada, com profissionais ${ }^{(5)}$. A construção e a validação de tecnologias, a serem incorporadas no cuidado e no ensino da enfermagem, alcançaram um crescimento exponencial nas últimas décadas com o propósito de promover a saúde de diversos grupos populacionais e facilitar a formação dos profissionais de saúde.

Nessa perspectiva, as pesquisas têm feito uso de estratégias dinâmicas e inovadoras que facilitam o acesso ao conhecimento, bem como o desenvolvimento da autonomia das pessoas. De maneira semelhante, os caminhos metodológicos adotados para o processo de validação são diversificados, a fim de verificar a capacidade das tecnologias em alcançar o objetivo proposto. Como exemplos, citam-se: ensino simulado, plataformas de cursos, vídeos, aconselhamento individual, apresentação em slides, manuais, impressos, cadernetas, jogos educativos, websites, cartilhas, softwares, teatro, entre outros, os quais foram criados e validados com uso de diferentes metodologias ${ }^{(4)}$.

Destarte, entende-se que a produção de estudos dessa natureza deva ser estimulada, em razão de seus benefícios, tanto para o ensino quanto para a saúde dos indivíduos e da população ${ }^{(5)}$. Porém, mesmo diante de sua relevância, não há na literatura um consenso quanto aos métodos mais indicados para o processo de validação científica de tecnologias educacionais. Por isso, é possível identificar diferentes referenciais teóricos e percursos metodológicos adotados pelas Teses e Dissertações da Enfermagem brasileira.

Outrossim, o estudo objetiva analisar as estratégias metodológicas utilizadas para a validação de tecnologias educacionais em teses e dissertações de Enfermagem no Brasil. Diante do objetivo, pretende-se responder às seguintes questões norteadoras: Qual o perfil das produções e das tecnologias educacionais validadas? Quais as estratégias metodológicas utilizadas para os processos de validação de tecnologias educacionais?

\section{MÉTODOS}

Trata-se de estudo do tipo documental bibliométrico de abordagem quantitativa. A bibliometria é uma técnica estatística que tem o objetivo de medir os índices de produção e disseminação do conhecimento científico em diferentes áreas, inclusive a Enfermagem. Essa também vem sendo utilizada para identificar os autores, os paradigmas da ciência, as metodologias e os periódicos mais utilizados ${ }^{(6)}$.

A busca pelos trabalhos foi realizada no catálogo de teses e dissertações da Coordenação de Aperfeiçoamento de Pessoal de Nível Superior (CAPES), do Ministério da Educação do Brasil (MEC), durante o mês de outubro de 2019. Não foram definidos filtros, como recorte de tempo, seleção de orientador, entre outros.

A estratégia de busca fez uso dos descritores: estudos de validação, tecnologia educacional, tecnologia e enfermagem, consultados nos Descritores em Ciências da Saúde (DeCS). Foram aplicados os seguintes cruzamentos com o operador booleano AND: (\#1 estudos de validação AND \#2 tecnologia AND \#3 enfermagem); e (\#1 estudos de validação AND \#4 tecnologia educacional AND \#3 enfermagem). 
O critério de inclusão correspondeu às pesquisas desenvolvidas pela enfermagem e que abordassem a validação de tecnologias educacionais com o objeto de estudo. Foram excluídas as teses e dissertações duplicadas, aquelas que validaram tecnologias assistenciais e não educativas, bem como as que não estavam disponíveis na íntegra. Por isso, uma vez selecionados trabalhos com publicação indisponível, foi realizado contato com os autores, via endereço eletrônico, a fim de obter o manuscrito na íntegra. Foram consideradas como perdas as teses e dissertações cujos autores não responderam ao contato ou optaram pela não disponibilidade do texto.

Após a obtenção do trabalho completo, foi realizada sua leitura sistemática, com o intuito de coletar as informações pertinentes ao estudo, processo que decorreu pela análise em pares, executada, concomitantemente, por quatro enfermeiros, discentes do Programa de Pósgraduação em Enfermagem da Universidade Federal do Rio Grande do Norte (UFRN). Essa dinâmica possibilitou que, em caso de dúvida, um dos pesquisadores pudesse realizar consulta aos demais autores.

Assim, com a estratégia de busca, obteve-se o total de 485 estudos, dos quais 87 foram excluídos por duplicação. Em seguida, procedeuse com a leitura de 398 resumos, sendo excluídos 220 por não atenderem aos critérios de elegibilidade. Desses, 21 estudos estavam indisponíveis na íntegra, sendo realizado contato com os autores por meio de endereço eletrônico. Dois autores responderam ao contato, por meio do qual um disponibilizou o material solicitado, e o outro justificou a impossibilidade de prover o manuscrito em arquivo on-line. Ao final da seleção, a amostra compreendeu o total de 156 teses e dissertações, conforme Figura 1.

Figura 1 - Fluxograma da coleta das teses e dissertações no catálogo da CAPES. 2019.

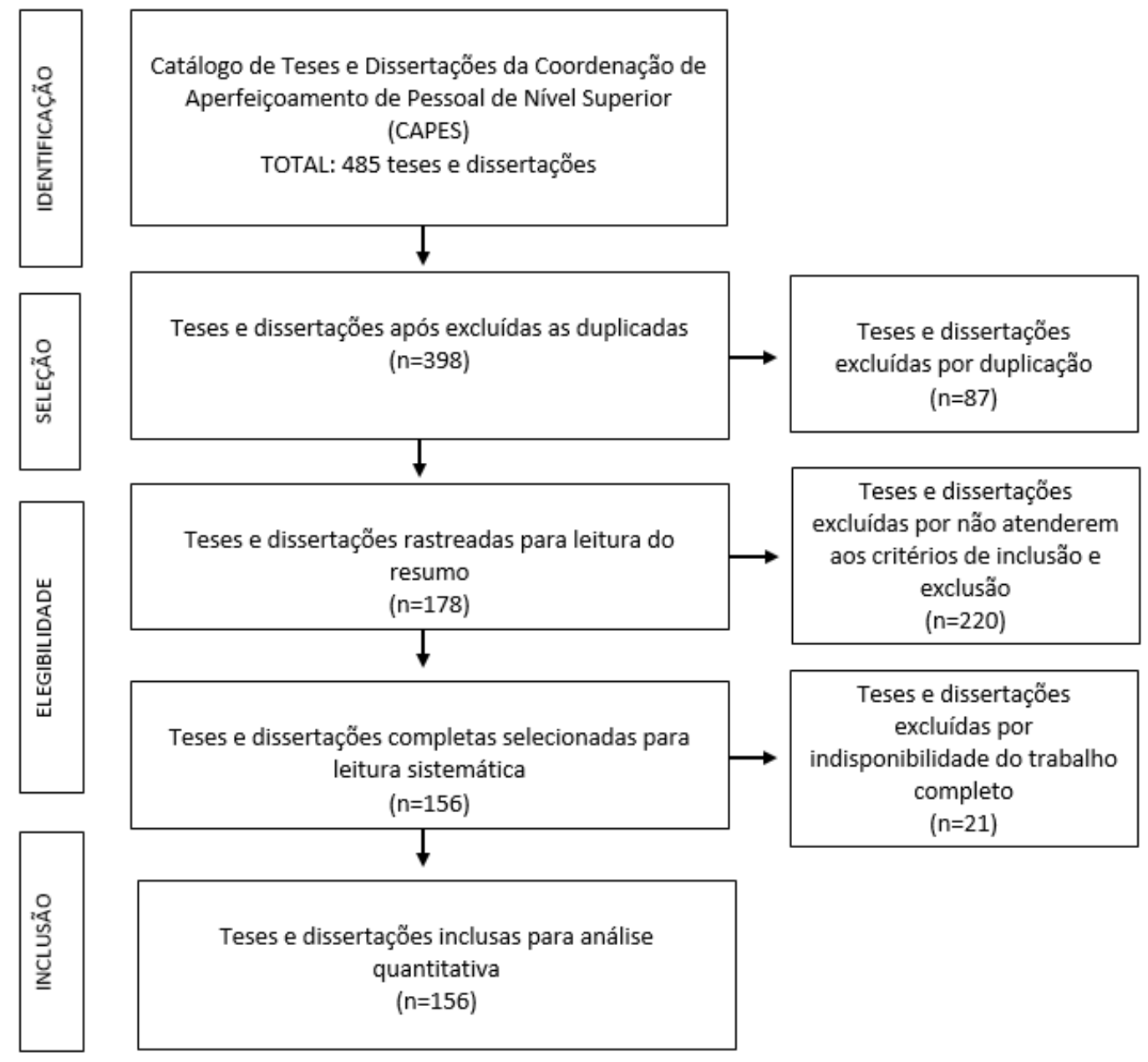

Fonte: Dados coletados pelos autores.

A leitura crítica dos estudos selecionados foi guiada por um banco de dados, elaborado pelos autores com o Microsoft Excel, o qual continha as variáveis de interesse do estudo. As variáveis 
relacionadas ao perfil das Teses e Dissertações foram: origem, ano e região geográfica; as referentes ao perfil das tecnologias educacionais foram: finalidade, área de conhecimento da enfermagem, tipologia e referencial utilizado para a produção. Quanto às estratégias do processo de validação das tecnologias educacionais, investigaram-se as seguintes variáveis: referencial metodológico, número de juízes especialistas e do público-alvo, critérios de seleção dos juízes, número de rodadas Delphi, instrumentos utilizados, valor mínimo de Índice de Validação de Conteúdo (IVC), tipo de estudo realizado com o público-alvo e técnicas de análise dos dados.

O conteúdo do banco de dados, em relação ao perfil das produções, das tecnologias educacionais e das estratégias de validação, foi analisado com uso do Statistical Package for the Social Sciences (SPSS) 21.0 para o cálculo da estatística descritiva com frequência relativa e absoluta. Os dados foram apresentados de forma descritiva por meio de gráficos, com o objetivo de facilitar a compreensão dos resultados.

O conteúdo global das produções, com vista a criar um corpus textual, foi analisado pelo uso do IRaMuTeQ 0.7 alpha 2. A matriz-base para produção do corpus foi o resumo das metodologias de cada trabalho. O software utilizado avalia a ocorrência dos termos, em trechos de 60 palavras, em média e forma agrupamentos dos fenômenos mais relevantes do trecho. Os dados relativos à análise lexical foram apresentados em forma de figuras, do tipo gráfico e nuvem de palavras. Tal análise visa atender à lei de zipt, a terceira lei da bibliometria, a qual relaciona a frequência de palavras utilizadas na área de pesquisa, de modo a identificar nomenclaturas para os fenômenos ou variáveis de destaque ao estudo. Infere-se que uma pequena quantidade de palavras pode ser utilizada em grande frequência, indicando o assunto principal do documento ${ }^{(6)}$.

\section{RESULTADOS E DISCUSSÃO \\ Perfil das Produções e Tecnologias Educacionais Validadas}

A maior porcentagem das produções 114 $(73,1 \%)$ correspondeu a pesquisas oriundas de dissertações, seja mestrado acadêmico 81 (51,9\%) ou profissional $33(21,2 \%)$, enquanto $42(26,9 \%)$ foram oriundas de teses. Observou-se crescimento exponencial, ao longo das duas últimas décadas, iniciando em 2011, com oito trabalhos, até alcançar o número de 45 em 2018, como pode ser observado na Figura 2.

Figura 2 - Teses e dissertação sobre validação de tecnologias educacionais publicadas por ano. 2019.

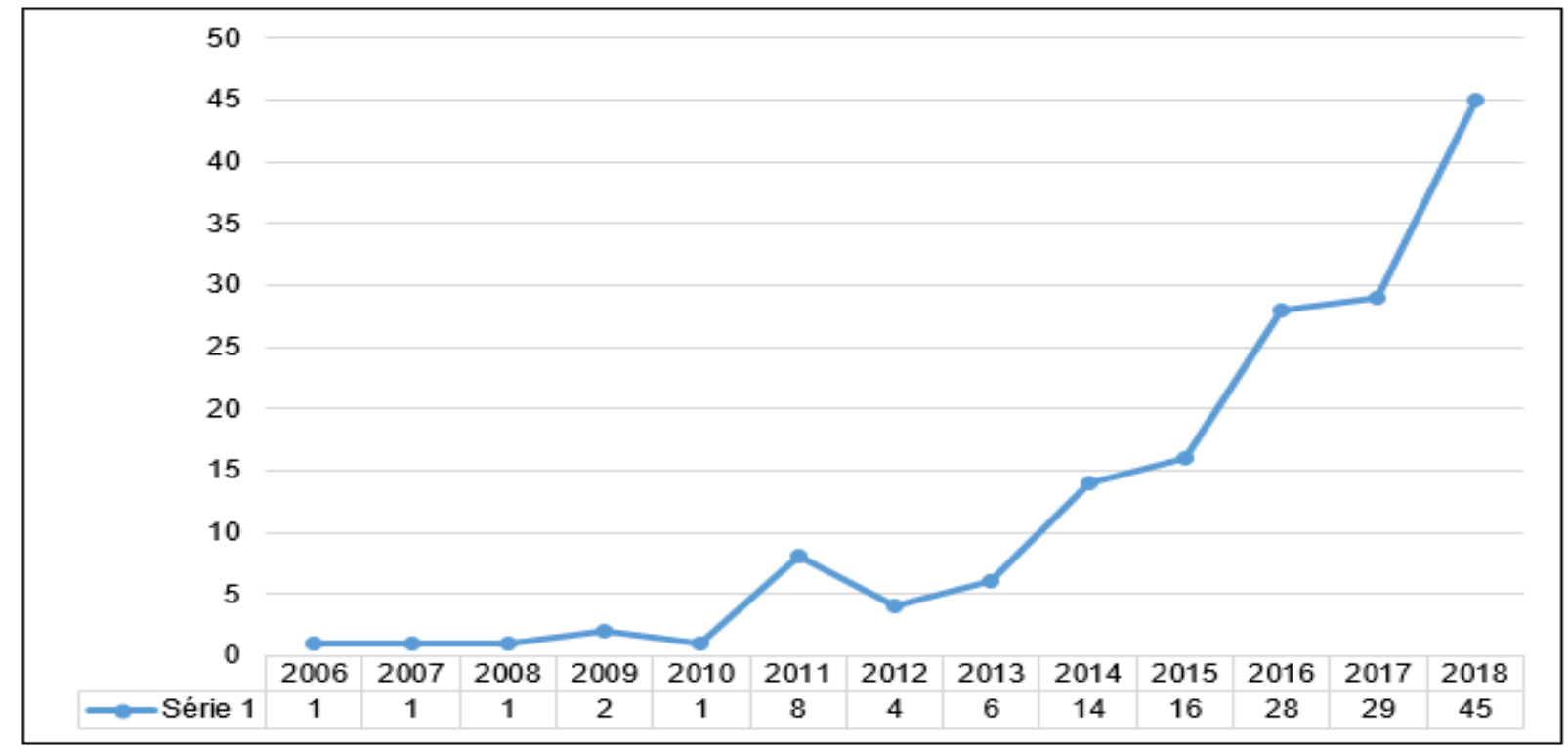

Fonte: Dados coletados pelos autores.

O avanço do desenvolvimento tecnológico da sociedade influencia não apenas os modos de produção e reprodução social, mas, principalmente, exerce impacto sobre a produção do conhecimento de diferentes disciplinas, como é o caso da Enfermagem. Isso, porque as transformações mediadas pelo aparato tecnológico na vida das pessoas, no fazer e no ensino em saúde, despontam no surgimento de necessidades que outrora eram inexistentes. 
Como reflexo, a criação de programas de pós-graduação latu sensu e a formação de grupos de pesquisa em enfermagem, com foco na tecnologia, dentre suas linhas de pesquisa, têm apresentado ritmo exponencial de crescimento nas últimas décadas. Esse contexto que impulsionou a formação de mestres e doutores na Enfermagem permitiu a presença, cada vez maior, desse profissional nos grupos de pesquisa em todo o País ${ }^{(7)}$. Não por acaso, verificou-se, neste estudo, o desenvolvimento crescente de dissertações 114 $(73,1 \%)$ e teses $42(26,9 \%)$, no período entre 2011 e 2018, as quais, em geral, vinculam-se a grupos e linhas de pesquisa que têm como foco o desenvolvimento tecnológico.

Ao considerar a região geográfica das produções, observa-se diferença na distribuição das produções, assim como representado na Figura 3.

Figura 3 - Distribuição das teses e dissertações por região de origem. 2019.

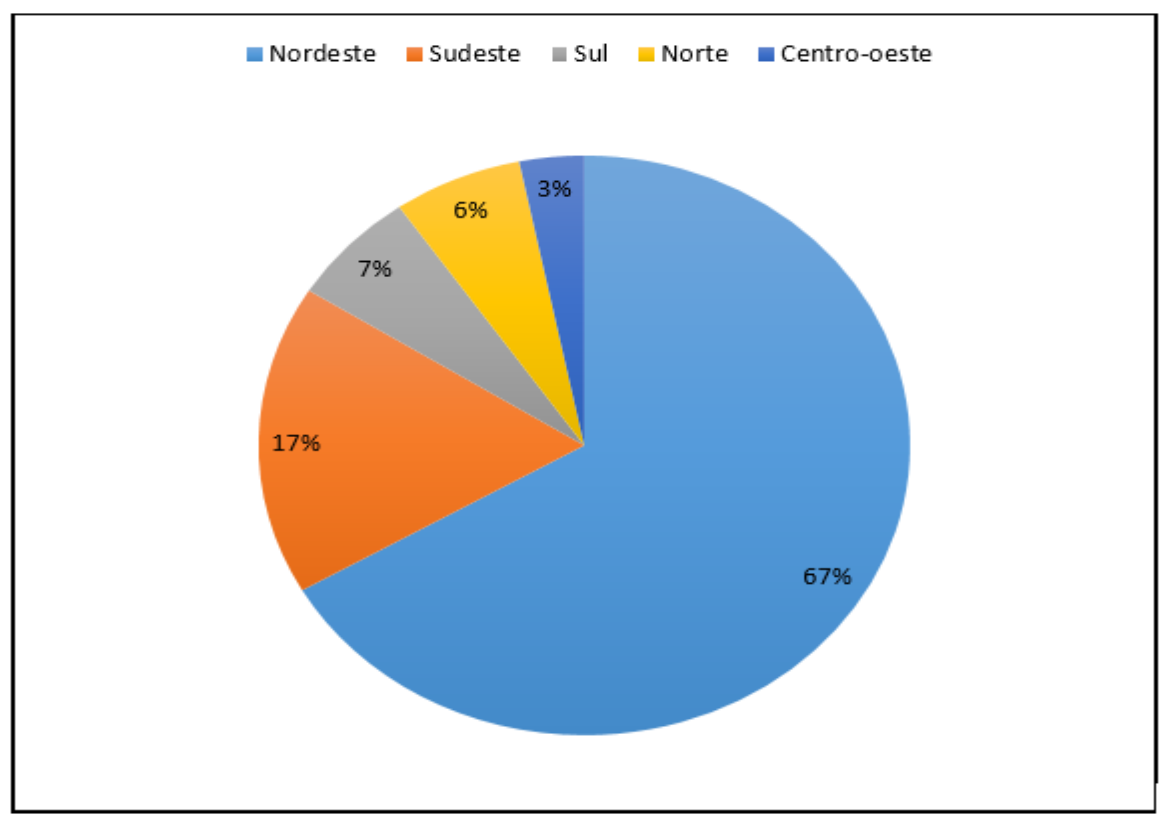

Fonte: Dados coletados pelos autores.

De forma detalhada, instituições como a Universidade Federal do Ceará (UFCE), Universidade Estadual do Ceará (UECE), Universidade de Fortaleza (UNIFOR), Universidade de São Paulo (USP) e a Universidade Federal do Pernambuco (UFPE) foram as principais responsáveis pelo desenvolvimento de pesquisas sobre o tema, representando, respectivamente, $54(34,6 \%), 17(10,9 \%), 14(9,0 \%), 12(7,7 \%)$ e 9 (5,8\%). A Universidade Federal Fluminense (UFF) e a Universidade do Amazonas (UFAM) realizaram 5 $(3,2 \%)$ estudos. Também, a Universidade Federal do Piauí (UFPI), Universidade Federal do Espírito Santo (UFES), Universidade Federal do Rio Grande do Norte (UFRN) e a Universidade Federal de Santa Maria (UFSM) tiveram a mesma porcentagem de trabalho, com o total de 4 (2,6\%). A Universidade Federal de Santa Catarina (UFSC) registrou $3(2,0 \%)$ estudos. Outras instituições, como a Universidade Franciscana (UFN), Universidade de Brasília (UNB), Universidade do Estado do Pará (UEPA) e a Fundação Santa Casa de Misericórdia do Pará (FSCMP) desenvolveram 2 $(1,3 \%)$, cada uma delas. Com apenas $1(0,6 \%)$ trabalho realizado estão as seguintes instituições: Universidade Federal de São Paulo (UNIFESP), Universidade Federal de Ciências da Saúde de Porto Alegre (UFCSPA), Universidade Federal de Sergipe (UFS), Universidade Federal de Mato Grosso (UFMT), Universidade Estadual Paulista (UNESP), Universidade Federal de Alagoas (UFAL), Universidade Federal da Paraíba (UFPB), Universidade Federal do Rio de Janeiro (UFRJ), Universidade Estadual de Maringá (UEM), Universidade do Vale do Rio dos Sinos (UNISINOS), Universidade Internacional da Lusofania Afrobrasileira (UNILAB), Universidade de Cruz da Alta 
(UNICRUZ) e Universidade Federal de Alfenas (UNIFAL).

A constatação de que a origem dos estudos, que versam sobre as $T E$, está concentrada na região Nordeste (67\%), coloca-se como um contraponto. Em estudo realizado sobre a produção científica da Enfermagem brasileira, foi possível verificar que o Sudeste desponta como a região com maior número de artigos publicados ${ }^{(8)}$ e com a maior proporção de grupos de pesquisa que investigam a temática da tecnologia ${ }^{(7)}$. Portanto a discrepância entre a proporção da produção de teses e dissertações por local de origem e a proporção de publicações de artigos científicos, segundo a localidade, remete à reflexão sobre a necessidade de se promover maior visibilidade, expressão nacional e internacional do conteúdo intelectual produzido pelas universidades nordestinas.

No que diz respeito às tecnologias educacionais validadas, 113 (72,4\%) foram consideradas tecnologias para a educação em saúde com a comunidade, $22(14,1 \%)$ para a educação no ensino técnico ou superior e 21 $(13,5 \%)$ para educação continuada com profissionais de saúde. Assim observa-se o crescente empenho na construção e validação de tecnologias voltadas para educação em saúde com a comunidade.

A expressividade de projetos, que pleiteiam o desenvolvimento dessa tecnologia, justifica-se pelos benefícios que as TE propiciam à população, como a melhoria na qualidade de vida, por meio da adoção de comportamentos saudáveis, ao passo que desenvolve a autonomia e o empoderamento dos sujeitos ${ }^{(9)}$.

Em relação às áreas de conhecimento, observou-se a seguinte distribuição: 60 (38,5\%) na área de enfermagem médico-cirúrgica, 32 (20,5\%) em enfermagem pediátrica, $20(12,8 \%)$ em enfermagem obstétrica, $20(12,8 \%)$ na área de métodos e técnicas de ensino, $14(9,0 \%)$ em ginecologia e obstetrícia, além de $10(6,4 \%)$ em enfermagem de saúde pública.

Embora tenha sido identificada uma variedade de áreas de conhecimento da enfermagem aplicada à tecnologia, sobressaíramse as temáticas relacionadas à enfermagem médico-cirúrgica $(38,5 \%)$, pediátrica $(20,5 \%)$ e obstétrica $(12,8 \%)$. Essa tendência converge para o que preconiza a Política Nacional de Ciência, Tecnologia e Inovação em Saúde (PNCTIS), por meio da Agenda Nacional de Prioridades de
Pesquisa em Saúde (ANPPS) e pelas Prioridades de Pesquisa em Enfermagem (PPE) ${ }^{(10)}$.

As PPE, discutidas desde 2011, tem como foco ajustar a pesquisa dessa área de atuação, em direção ao cuidado da enfermagem, à população e às problemáticas nacionais. Com isso, dentre seus 11 eixos temáticos, encontram-se a investigação clínica em enfermagem, o cuidado de enfermagem à saúde da mulher e o cuidado de enfermagem à saúde da criança e do adolescente como temas primordiais a serem pesquisados ${ }^{(10)}$.

Os artefatos tecnológicos validados foram: 66 trabalhos $(42,3 \%)$ desenvolveram impressos; $24(15,4 \%)$ softwares ou aplicativos; $22(14,1 \%)$ vídeos; 19 (12,2\%) Ambientes Virtuais de Aprendizagem (AVA); $10(6,4 \%)$ simuladores; cinco $(3,2 \%)$ tecnologia assistida; quatro $(2,6 \%)$ jogos; quatro $(2,6 \%)$ áudio ou podcast; 1 (um) $(0,6 \%)$ brinquedos e histórias em quadrinhos; e 1 (um) $(0,6 \%)$ tecnologias associadas (impressos, vídeo e programas).

Observa-se que as pesquisas fizeram uso de produtos tecnológicos diversos, com destaque para os materiais impressos, softwares ou aplicativos, vídeos e os AVA. Tradicionalmente, algumas modalidades de TE têm sido empregadas, mais frequentemente, pelos enfermeiros, como as cartilhas, impressos, manuais, álbuns educativos, vídeos, jogos e outros recursos audiovisuais ${ }^{(5,11)}$. Sugere-se que a eleição dos instrumentos citados pode ser influenciada pela praticidade, facilidade de acesso e ludicidade que eles podem garantir ao aprendizado, tornando-os mais participativos e integrados com a realidade das pessoas.

O tipo de estudo predominante foi o metodológico, citado por $120(76,9 \%)$ autores. Também foram denominados estudos de desenvolvimento tecnológico, em 17 trabalhos $(10,9 \%)$, multimétodos em $11(7,1 \%)$ e quaseexperimental por oito autores $(5,1 \%)$.

Para que as tecnologias possam ser utilizadas pelos profissionais de saúde e aplicadas junto ao público-alvo, faz-se necessário que sejam submetidas à validação. Esse processo compreende o grau de evidência empírica e racionalização teórica que apoia a adequabilidade das inferências, mediante a aplicação de modos avaliativos e testes $^{(11)}$. Em outras palavras, confere validade científica, segurança e uniformização dos conteúdos abordados, de modo a alcançar os objetivos aos quais se propõem.

No que tange à produção das tecnologias educacionais validadas, observou-se a adoção de referenciais, como: design instrucional, 
contextualizado em 1 (um) (0,6\%) estudo; design de interação participativo, centrado no usuário, em 1(um) (0,6\%); user-centered design em 1 (um) $(0,6 \%)$; Galvis-Panqueva em dois (1,2\%); e de Echer em cinco $(3,2 \%)$ trabalhos. Também foram mencionadas diferentes teorias, como: teoria do autocuidado, de Dorothea Orem, em sete $(4,5 \%)$ estudos; autoeficácia, de Brandura, em três (1,9\%); tradução do conhecimento em 1(um) $(0,6 \%)$; e transpessoal em 1 (um) $(0,6 \%)$. Além de serem utilizados aportes pedagógicos, a exemplo: da teoria de interação social de Vygotsky, em cinco $(3,2 \%)$ estudos; da abordagem dialógica de Paulo Freire, em dois (1,2\%); e do construtivismo de Piaget, em 1(um) (0,6\%) trabalho.

Ademais, foi citada a utilização de modelos na construção das tecnologias, quais sejam: modelo de atividade de vida de Roper, Logan e Tierney, em 1(um) (0,6\%) pesquisa; modelo de crenças em saúde, em 1 (um) (0,6\%); e o modelo para concepção e desenvolvimento de material educativo digital de Falkemback, em seis $(3,8 \%)$ estudos.

\section{Estratégias de Validação}

Quanto ao processo de validação, 19 $(12,1 \%)$ trabalhos adotaram o modelo de validação de Pasquali, como referencial para a sua condução. Para o processo de validação, as pesquisas em saúde têm adotado o modelo de Pasquali, a despeito de que $12,1 \%$ dos trabalhos analisados por este estudo o consideram como referencial metodológico. Por ser amplamente utilizado na Psicologia, para a elaboração de escalas psicométricas, adaptações do modelo às peculiaridades do desenvolvimento das tecnologias têm sido realizadas nos estudos, porém de modo a manter o conjunto de procedimentos que constituem os polos teórico, empírico (experimental) e analítico ${ }^{(12)}$

Em termos metodológicos e de forma detalhada, verificou-se que, em 148 (94,9\%) produções, participaram juízes especialistas da área da saúde e, em 81 (51,9\%), juízes de outras áreas como designer, marketing, tecnologia da informação, comunicação e pedagogia. A participação do público-alvo se deu em 94 (60,3\%) produções. Sobretudo, 60 (38,5\%) estudos foram validados por consenso, envolvendo somente juízes técnicos; 84 (53,8\%), por meio da validação de concordância, cujo processo envolve avaliação dos juízes e da população-alvo; e 12 (7,7\%) foram submetidos à validação clínica apenas com a população-alvo.
A literatura especializada cita a validação por concordância (apreciação por juízesespecialistas e pelo público-alvo) e a validação por consenso (apreciação apenas por expertises) como caminhos possíveis, sendo o estímulo à participação dos sujeitos, que compõem o público-alvo, uma tendência crescente ${ }^{(4)}$. Assim, observou-se, neste trabalho, que 84 (53,8\%) pesquisas adotaram a validação por concordância, com ênfase na participação do público-alvo, assim como recomendado.

O número de juízes teve ampla variação, com mínimo de três especialistas e máximo de 75 , obtendo-se uma média de 16 participantes. Há controvérsias sobre o número e os critérios de seleção utilizados para definição dos juízesespecialistas. Em decorrência, ficou evidente a variação de referências utilizadas para os critérios de seleção dos juízes e do número amostral. Contudo existe o consenso de que eles devem estar claros, bem como para sua definição, devem ser avaliadas as características do instrumento utilizado, qualificação e disponibilidade dos profissionais necessários ${ }^{(11)}$.

Para seleção dos juízes-especialistas, os critérios adotados foram: Fehring (1994), em 63 (43\%) estudos; elaborados pelo autor em 53 (35,5\%); Jasper (1994), em 16 (10,8\%); Barbosa (2008), em 10 (6,7\%); e, dentre outros autores, em seis trabalhos $(4,0 \%)$. Nos estudos que fizeram uso da validação com juízes-especialistas, a técnica Delphi foi empregada em 129 (87,2\%), com uma rodada de avaliação, em 15 (10,1\%), com duas rodadas e em quatro $(2,7 \%)$, com três rodadas.

Uma vez identificados os especialistas participantes e realizado contato para fornecer as informações, foi possível a realização de duas rodadas da técnica Delphi, a fim de atingir o IVC acima do considerado(5). Apesar de ser recomendada a realização de duas rodadas Delphi, $88,2 \%$ dos trabalhos investigados por esta revisão alcançaram um resultado satisfatório em apenas um ciclo de Delphi.

Os tipos de estudo realizados com o público-alvo foram: $47(30,2 \%)$ do tipo exploratórios e descritivos, seguidos de $24(15,4 \%)$ quase-experimentos e sete $(4,5 \%)$ ensaios clínicos, nos quais a amostra variou entre 1 a 596 sujeitos, com média de 54 participantes. Verificou-se, assim, que predominaram os estudos exploratórios e descritivos $(30,2 \%)$, os quais possuem nível de $\mathrm{V}$ (cinco) de evidência científica. Esse fato sugere a necessidade de os enfermeiros empreenderem pesquisas mais robustas, em 
termos de evidência, quando compatíveis com os objetos de estudos, a exemplo de pesquisas quase-experimentais e ensaios clínicos.

Dos instrumentos utilizados, 98 (62,8\%) foram validados e adaptados ao contexto da tecnologia em questão. Mas, também, em 58 (37, $2 \%$ ) os autores optaram por elaborar seus instrumentos. O contato com os participantes, juízes ou sujeitos do público-alvo, foi realizado de forma on-line e por abordagem individual em 82 (52,6\%), apenas on-line em $44(28,2 \%)$ ou por abordagem individual em 30 (19,2\%) trabalhos.

Para a etapa de coleta de dados, é recorrente que os autores façam uso de instrumentos e questionários previamente validados, a exemplo do Suitability Assessment of Materials (SAM), mas que sejam adaptados, de acordo com as especificidades do estudo em questão, principalmente, no que diz respeito às características da população ${ }^{(13)}$.

A análise dos dados foi realizada com técnicas variadas, com predomínio da estatística descritiva, na qual todos os trabalhos utilizaram a aplicação de frequências relativas e absolutas. Uma das medidas empregadas com maior frequência foi o IVC, aplicado em 127 (81,4\%) análises, cujos valores de referência foram diversificados, com maior emprego do valor mínimo de 0,80 em 71 (55,9\%) estudos. A média do IVC, obtida pelas pesquisas analisadas, foi de 0,90 , com mínimo de 0,70 e máximo de 1,00 . A abordagem qualitativa foi utilizada em 112 (71,7 \%) trabalhos, como estratégia para a análise das sugestões dos juízes, especialmente, nas situações em que o IVC obteve valor abaixo da referência adotada.

O IVC corresponde a um método que mede, a partir de uma escala tipo likert, a porcentagem de juízes que concordam sobre determinados aspectos e itens da tecnologia, obtendo-se uma valoração quantitativa de sua validade. Por esse motivo, tem sido amplamente recomendado e utilizado nas pesquisas em saúde, no entanto diferentes valores mínimos de referência são adotados pelos estudos. Apesar da variedade, uma taxa inferior a 0,78 não é recomendada, sendo o valor de 0,80 o maior utilizado, assim como verificado nos estudos desta pesquisa bibliométrica $^{(13)}$.

Além do IVC, outras medidas foram utilizadas no processo de validação, tais como: alfa de cronbach, em 14 (8,9\%) pesquisas; índice de legibilidade de flesch, em sete $(4,4 \%) ;$ SAM, em seis $(3,8 \%)$; índice kappa, em quatro $(2,5 \%)$; e o Coeficiente de Correlação Interclasses (CCI), em quatro (2,5\%). Adicionalmente, nos estudos que envolveram a validação clínica, análises inferenciais foram empregadas, a exemplo dos testes qui-quadrado, em 17 (10,8\%) trabalhos; t student, em 15 (9,6\%); wilcoxon, em 13 (8,3\%); mann-whitney, em 11 (7,0\%); ANOVA, em sete $(4,4 \%)$; mcNemar, em seis $(3,8 \%)$; fisher, em seis $(3,8 \%)$; pearson, em cinco $(3,2 \%)$; tukey, em quatro (2,5\%); kolmogorov-smivorv, em dois $(1,2 \%)$; razão de chance, em dois $(1,2 \%)$; e friedman, em 1 (um) $(0,6 \%)$ estudo.

\section{Corpus Textual}

Quanto à terceira lei de zipt, foi possível identificar que, nas 156 produções, foram utilizadas 3.627 palavras, das quais 686 eram termos distintos e 422 (61,5\%) surgiram uma única vez (hapax). Portanto, de forma inversamente proporcional, houve menor número de palavras que foram citadas com maior frequência, enquanto um significativo número de palavras citadas poucas vezes.

Na Figura 4, observam-se, em destaque na nuvem de palavras, os termos com maior frequência de citação: validação (151), juiz (91) e estudo metodológico (81), enquanto as palavras subjacentes, a exemplo de conteúdo (76), especialistas (72), público-alvo (54) e aparência (42) tiveram menor frequência. 
Figura 4 - Nuvem de palavras que compõem o corpus textual das teses e dissertações. 2019.

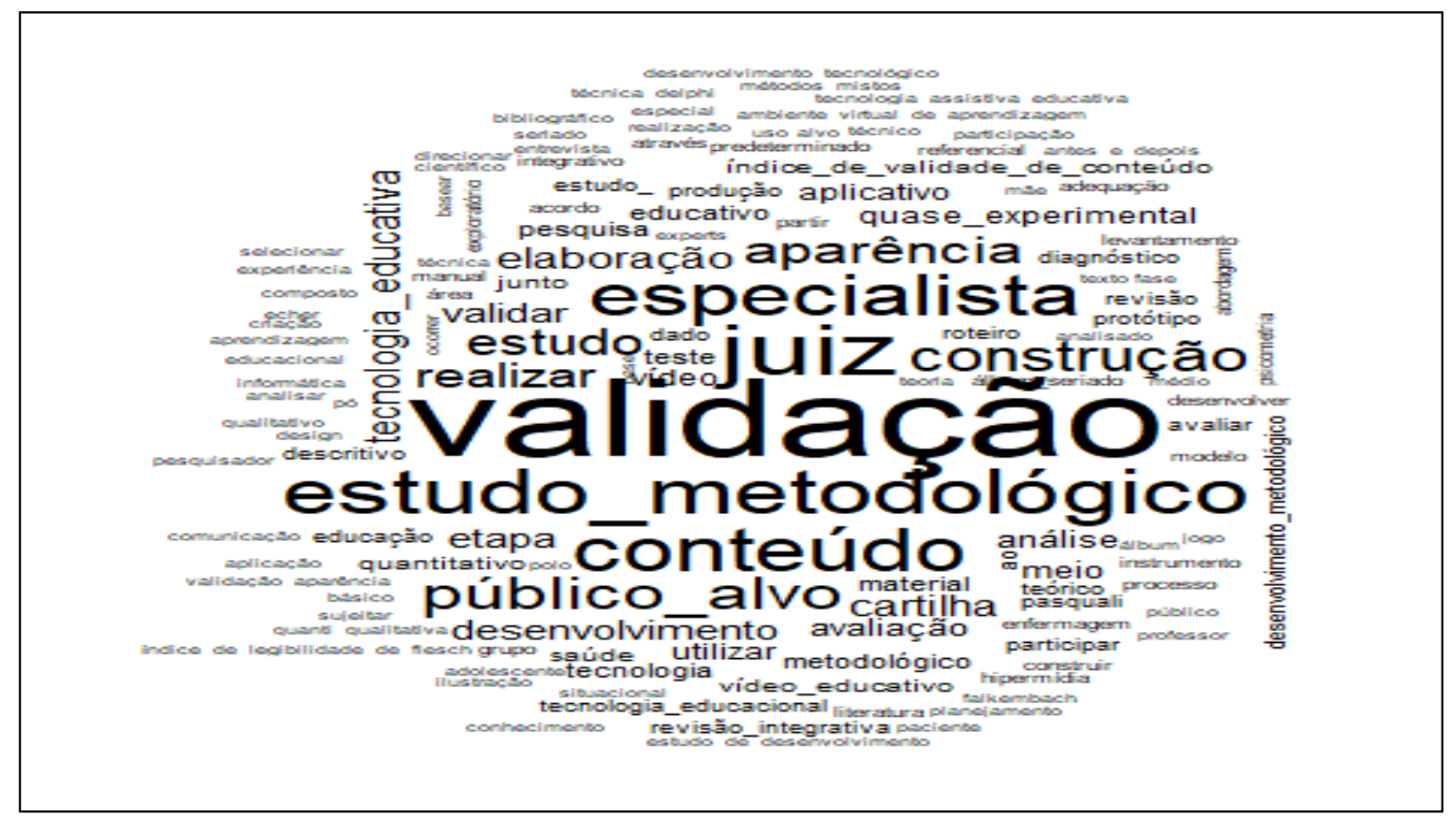

Fonte: Dados coletados pelos autores.

Não por acaso, a palavra validação foi o termo com maior ocorrência (151) na análise de Zipt, seguida dos elementos que a compõem, como o tipo de estudo metodológico, a participação dos juízes especialistas e do públicoalvo e a validação do conteúdo e aparência.

Em conjunto com o modelo de validação, a metodologia mais comumente adotada tem sido os estudos metodológicos $(76,9 \%)$, visto que possibilitam a validação das $\mathrm{TE}$, quanto ao conteúdo, por juízes especialistas e, quanto à aparência, pelo público-alvo. Segundo Nietshe, Texeira e Medeiros (2017), garantir a efetivação dessas etapas e, principalmente, possibilitar a participação de sujeitos que compõem a população-alvo é um dos grandes desafios desse campo de estudo(5).

Diante disso, entende-se que os estudos metodológicos são investigações que visam à obtenção e à organização dos dados para validar e avaliar, rigorosamente, ferramentas e métodos de pesquisa. Sua meta é a elaboração de instrumentos confiáveis, precisos e aplicáveis em diferentes realidades, por diferentes pesquisadores e pessoas, os quais lidam com fenômenos complexos, como comportamentos ou a saúde dos indivíduos, os quais têm sido amplamente utilizados pela Enfermagem, em diversas áreas de conhecimento, com o propósito de desenvolver e validar diferentes tecnologias como, por exemplo, as $\mathrm{TE}^{(14)}$.

\section{CONCLUSÃO}

As estratégias de validação de tecnologias educacionais são múltiplas e suscitam a sua potencialidade em articular a educação em saúde com a tecnologia, métodos, técnicas e referenciais teóricos de pesquisa. Em suma, as pesquisas que objetivaram validar tecnologias dessa natureza passaram a ter um crescimento considerável, a partir de 2008, sendo o Nordeste a região do País que mais desenvolve esse estudo. $O$ tipo de estudo predominante foi o metodológico, e os métodos empregados incluíram a validação por juízes especialistas da área da saúde e a validação junto ao público-alvo. Para esse último, comumente foram realizados estudos exploratórios e descritivos.

A análise dos dados, por meio da estatística descritiva e inferencial, esteve presente em quase todos os trabalhos, assim como o IVC também foi empregado com significativa frequência. Ressaltase a diversidade de referenciais teóricos utilizados, o que demonstra a dificuldade dos pesquisados em estabelecer um consenso quanto ao embasamento teórico mais adequado para o processo de validação.

O mapeamento apresentado propiciou a visibilidade da produção científica acerca da validação de tecnologias educacionais, indicando um conhecimento com distribuição desigual no que tange à região onde se produz tal capital intelectual. $O$ estudo contribuiu para o avanço do 
conhecimento da enfermagem, ao clarificar os métodos utilizados pelos enfermeiros, nas pesquisas de validação de tecnologia, o que poderá colaborar para o desenvolvimento de novos estudos.

Como limitações, pela indisponibilidade de teses e dissertações que, inicialmente, fizeram parte da amostra, não foi possível analisar toda a literatura produzida e publicada no diretório CAPES. Por fim, recomenda-se o desenvolvimento de estudos que possam mapear a validação de outros tipos de tecnologias.

\section{REFERÊNCIAS}

1- Salbego C, Nietsche EA, Teixeira E, GirardonPerlini NMO, Selvagem CF, Ilha S. Tecnologias cuidado-educativas: Um conceito emergente da práxis do enfermeiro em contexto hospita'lar. Rev Bras Enferm. 2018;71 (supl 6):2666-74. DOI: 10.1590/0034-7167-2017-0753

2- Salvador PTCO, Rodrigues CCFM, Lima KTN, Alves KYA, Santos VEP. Uso e desenvolvimento de tecnologias para o ensino apresentados em pesquisas de enfermagem. Rev Rene 2015;16(3):442-50. DOI: $10.15253 / 2175-$ $\underline{6783.2015000300018}$

3- Carvalho DS, Silva AGI, Ferreiraa SRM, Braga LC. Elaboration of an educational technology for ostomized patients: Peristomal skin care. Rev Bras Enferm. 2018;72(2):427-34. DOI: 10.1590/00347167-2016-0024

4- Afio ACE, Balbino AC, Alves MDS, Carvalho LV, Santos MCL, Oliveira NR. Analysis of the concept of nursing educational technology applied to the patient. Rev Rene 2014;15(1):158-65. DOI: 10.1590/0034-7167-2016-0024

5- Nietshe EA, Teixeira E, Medeiros HP. Tecnologias cuidativo-educacionais: Uma possibilidade para o empoderamento do/a enfermeiro. Porto Alegre: Moria; 2017.

6- Quevedo-Silva F, Santos EBA, Brandão MM, VL. Estudo bibliométrico: Orientações sobre sua aplicação. REMark 2016;15(2):245-62. DOI: 10.5585/remark.v15i2.3274

7- Erdmann AL, Peiter CC, Lanzoni GMM. Grupos de pesquisa em enfermagem no Brasil: Comparação dos perfis de 2006 e 2016. Rev Gaúcha Enferm. 2017;38(2):1-7. DOI: 10.1590/1983-1447.2017.02.69051

8- Melo ECA, Enders BC. Tecnologias educacionais: Produção científica da enfermagem brasileira. In: Anais do 170 Seminário Nacional de Pesquisa em Enfermagem, 2013; Natal. Natal: ABEn; 2013. p. 942.
9- Pereira ELC, Sanguino GZ, Ronchi TS, Previanato GF, Jaques AE, Baldissera VDA, et al. Tecnologias educativas gerontogeriátricas nas diferentes temáticas de saúde: Uma revisão integrativa. Rev Enferm Cent-Oeste Min. 2019;9:18. DOI: $10.19175 /$ recom.v9i0.2768

10- Oliveira DC. Nursing research priorities and research lines: an on-going discussion. Rev Enferm UERJ 2014;22(5):712-6. DOI: 10.12957/reuerj.2014.12771

11- Silva DML, Carreiro FA, Mello R. Educational technologies in nursing assistance in health education: Integrat ingreview. J Nurs UFPE 2017;11(Suppl):1044-52. DOI: 10.5205/19818963-V11I2A13475P1044-1051-2017

12- Medeiros RKS, Ferreira Júnior MA, Pinto DPSR, Vitor AF, Santos VEP, Barichello E. Modelo de validação de conteúdo de Pasquali nas pesquisas em Enfermagem. Rev Enf Ref. 2015;4(4):127-35. DOI: 10.12707/RIV14009

13- Alexandre NMC, Coluci MO. Validade de conteúdo nos processos de construção e adaptação de instrumentos de medidas. Ciênc Saúde Coletiva 2011;16(7):3061-68. DOI: 10.1590/S1413-81232011000800006

14- Sabino LMM, Ferreira AMV, Mendes ERR, Joventino ES, Gubert FA, Penha JC, et al. Validação de cartilha para promoção da autoeficácia materna na prevenção da diarreia infantil. Rev Bras Enferm. 2018;71(suppl 3):1412-19. DOI: 10.1590/0034-7167-2017-0341

Nota: Não houve financiamento por agência de fomento

Recebido em: 09/07/2020

Aprovado em: 14/12/2020

Endereço de correspondência:

Alexandra do Nascimento Cassiano

Rua dos Cajueiros, 115, apto 406, bloco 1, Nova Parnamirim, Natal, Rio Grande do Norte, Brasil. CEP.: 59150-600.

E-mail: anc_enfa@hotmail.com 